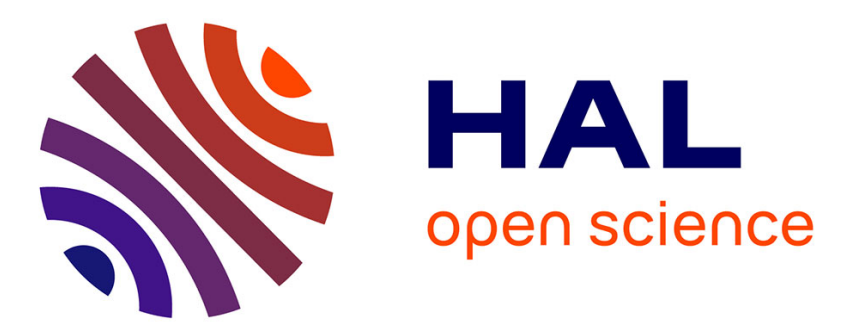

\title{
Transport equations for reaction rate in laminar and turbulent premixed flames characterized by non-unity Lewis number
}

Andrei N. Lipatnikov, Nilanjan Chakraborty, Vladimir Sabelnikov

\section{- To cite this version:}

Andrei N. Lipatnikov, Nilanjan Chakraborty, Vladimir Sabelnikov. Transport equations for reaction rate in laminar and turbulent premixed flames characterized by non-unity Lewis number. International Journal of Hydrogen Energy, 2018, 43, pp.21060-21069. 10.1016/j.ijhydene.2018.09.082 . hal-02315556

\author{
HAL Id: hal-02315556 \\ https://hal.science/hal-02315556
}

Submitted on 14 Oct 2019

HAL is a multi-disciplinary open access archive for the deposit and dissemination of scientific research documents, whether they are published or not. The documents may come from teaching and research institutions in France or abroad, or from public or private research centers.
L'archive ouverte pluridisciplinaire HAL, est destinée au dépôt et à la diffusion de documents scientifiques de niveau recherche, publiés ou non, émanant des établissements d'enseignement et de recherche français ou étrangers, des laboratoires publics ou privés. 


\title{
Transport equations for reaction rate in laminar and turbulent premixed flames characterized by non-unity Lewis number
}

\author{
Andrei N. Lipatnikov ${ }^{1}$, Nilanjan Chakraborty ${ }^{2}$, Vladimir A. Sabelnikov ${ }^{3,4}$ \\ ${ }^{1}$ Department of Mechanics and Maritime Sciences, Chalmers University of Technology, Gothenburg, 41296 Sweden \\ ${ }^{2}$ School of Mechanical and Systems Engineering, Newcastle University, Newcastle Upon Tyne NE1 7RU, United Kingdom \\ ${ }^{3}$ ONERA - The French Aerospace Laboratory, F-91761 Palaiseau, France \\ ${ }^{4}$ Central Aerohydrodynamic Institute (TsAGI), 140180 Zhukovsky, Moscow Region, Russian Federation
}

\begin{abstract}
Transport equations for (i) the rate $W$ of product creation and (ii) its Favre-averaged value $\widetilde{W}$ are derived from the first principles by assuming that $W$ depends solely on the temperature and mass fraction of a deficient reactant in a premixed turbulent flame characterized by the Lewis number Le different from unity. The right hand side of the transport equation for $\widetilde{W}$ involves seven unclosed terms, with some of them having opposite signs and approximately equal large magnitudes when compared to the left-hand-side terms. Accordingly, separately closing each term does not seem to be a promising approach, but a joint closure relation for the sum $\overline{T_{\Sigma}}$ of the seven terms is sought. For this purpose, theoretical and numerical investigations of variously stretched laminar premixed flames characterized by $L e<1$ are performed and the linear relation between $T_{\Sigma}$ integrated along the normal to a laminar flame and a product of (i) the consumption velocity $u_{c}$ and (ii) the stretch rate $\dot{s}_{w}$ evaluated in the flame reaction zone is obtained. Based on this finding and simple physical reasoning, a joint closure relation of $\overline{T_{\Sigma}} \propto \overline{\rho W \dot{s}}$ is hypothesized, where $\rho$ is the density and $\dot{s}$ is the stretch rate. The joint closure relation is tested against 3D DNS data obtained from three statistically 1D, planar, adiabatic, premixed turbulent flames in the case of a single-step chemistry and $L e=0.34,0.6$, or 0.8 . In all three cases, agreement between $\overline{T_{\Sigma}}$ and $\overline{\rho W \dot{s}}$ extracted from the DNS is good with exception of large $(\bar{c}>0.4)$ values of the mean combustion progress variable $\bar{c}$ in the case of $L e=0.34$. The developed linear relation between $\overline{T_{\Sigma}}$ and $\overline{\rho W \dot{s}}$ helps to understand why the leading edge of a premixed turbulent flame brush can control its speed.
\end{abstract}

Keywords: premixed turbulent combustion, mean reaction rate, Lewis number, turbulent flame speed, modeling, DNS

Declarations of interest: none

\section{Introduction}

The most important peculiarity of turbulent combustion of lean hydrogen-air mixtures consists of an abnormally large (when compared to hydrocarbon-air mixtures burned under similar conditions) ratio of turbulent, $S_{t}$, and laminar, $S_{L}$, flame speeds. This phenomenon is well documented in numerous experiments reviewed elsewhere [1-3], see also Refs. [4,5] as recent examples. The same phenomenon is also documented for lean turbulent flames of various $\mathrm{H}_{2} / \mathrm{CO} / \mathrm{CH}_{4} / \mathrm{O}_{2} / \mathrm{N}_{2}$ mixtures [6-11], but the magnitude of the effect is decreased when the mole fraction of hydrogen in the fuel blend is decreased by retaining the same equivalence ratio. 
In qualitative discussions, the considered phenomenon is commonly attributed to diffusive-thermal and preferential diffusion effects, which can strongly increase the local burning rate by significantly increasing the local temperature and equivalence ratio in highly curved and strained reaction zones, as discussed in details elsewhere [1-3]. The theory of such effects has yet been developed solely for the laminar premixed flames [12-14], with the focus of the theory being placed on the difference in the molecular diffusivity $D$ of the deficient reactant (e.g., hydrogen in a lean hydrogen-air mixture) and the heat diffusivity $\kappa$ of the mixture. This difference is commonly characterized with the Lewis number $L e=\kappa / D$, which is about 0.3-0.4 for very lean hydrogen-air mixtures and is larger, but is less than unity for lean $\mathrm{H}_{2} / \mathrm{CO} / \mathrm{CH}_{4} / \mathrm{O}_{2} / \mathrm{N}_{2}$ mixtures. Accordingly, the aforementioned abnormally large ratio of $S_{t} / S_{L}$ in lean hydrogen-air or $\mathrm{H}_{2} / \mathrm{CO} / \mathrm{CH}_{4} / \mathrm{O}_{2} / \mathrm{N}_{2}$ flames is typically discussed in terms of a strong increase in $S_{t}$ with decreasing $L e$ [1-3].

Although the aforementioned diffusive-thermal and preferential diffusion effects are widely recognized to be the governing physical mechanisms of the well-documented increase in $S_{t}$ with decreasing $L e$, the phenomenon remains to be one of the major poorly resolved fundamental issues in the combustion science. Indeed, quantitative prediction of a large ratio of $S_{t} / S_{L}$ at a low $L e$ still challenges the combustion community and the vast majority of numerical models of premixed turbulent flames, reviewed elsewhere [15-19], either disregard the influence of Lewis number on turbulent burning rate or invoke highly empirical fits.

The present work aims at filling this gap by (i) extending a recently introduced approach [20-22] to numerical modeling of premixed turbulent combustion to the case of $L e \neq 1$ and (ii) validating the extended approach against Direct Numerical Simulation (DNS) data obtained from premixed turbulent flames characterized by $L e<1$. The approach directly addresses transport equations for reaction rate $W$ and its Favre-averaged value $\widetilde{W}$, but has yet been restricted to the case of $L e=1[20$ 22]. It is worth remembering that averaging reaction rates subject to fluctuations in the local temperature and concentrations is on the most critical issue of the turbulent combustion theory even in the case of $L e \neq 1$ and the recently introduced transport equations for $W$ and $\widetilde{W}$ offer new opportunities to make a progress in this area.

The structure of the paper is as follows. In the next section, transport equations for $W$ and $\widetilde{W}$ are derived in the case of $L e \neq 1$. In the third section, the former equation is applied to various stretched laminar premixed flames. In the fourth section, based on analytical and numerical results obtained for the laminar flames, a joint closure relation for all terms on the Right Hand Side (RHS) of the transport equation for $\widetilde{W}$ is proposed to be used. The joint closure relation is validated by analyzing DNS data in the fifth section, followed by conclusions.

\section{Derivation}

Let us assume that the state of the mixture is characterized with (i) the combustion progress variable $c=1-Y / Y_{u}$ and (ii) the normalized temperature $\theta=\left(T-T_{u}\right) /\left(T_{a}-T_{u}\right)$, i.e., $\rho=\rho(c, \theta), W=W(c, \theta)$, etc. Here, $Y$ is the mass fraction of the deficient reactant, $T$ is the temperature, $T_{a}$ is the adiabatic combustion temperature (note that $\theta$ may be greater than unity if $L e<1), \rho$ is the mixture density, subscripts $u$ and $b$ designate unburned and burned mixture, respectively. The invoked 
assumptions are typical, e.g., in theoretical studies of stretched laminar flames [12-14]. The assumptions hold, e.g., in the adiabatic, low-Mach-number, single-step-chemistry ${ }^{1}$ case.

Applying a chain rule to express temporal and spatial derivatives of $W=W(c, \theta)$ as functions of temporal and spatial derivatives of $c$ and $\theta$

$$
\rho \partial_{t} W+\rho \mathbf{u} \cdot \nabla W=W_{c}\left(\rho \partial_{t} c+\rho \mathbf{u} \cdot \nabla c\right)+W_{\theta}\left(\rho \partial_{t} \theta+\rho \mathbf{u} \cdot \nabla \theta\right)
$$

and using the following transport equations

$$
\begin{gathered}
\rho \partial_{t} c+\rho \mathbf{u} \cdot \nabla c=\nabla \cdot(\rho D \nabla c)+\rho W \\
\rho \partial_{t} \theta+\rho \mathbf{u} \cdot \nabla \theta=L e \nabla \cdot(\rho D \nabla \theta)+\rho W
\end{gathered}
$$

to transform the terms in parentheses on the RHS of Eq. (1), we arrive at

$$
\begin{gathered}
\rho \partial_{t} W+\rho \mathbf{u} \cdot \nabla W=W_{c}[\nabla \cdot(\rho D \nabla c)+\rho W]+W_{\theta}[L e \nabla \cdot(\rho D \nabla \theta)+\rho W] \\
=\nabla \cdot\left(\rho D W_{c} \nabla c\right)-\rho D \nabla c \cdot \nabla W_{c}+\rho W W_{c}+L e \nabla \cdot\left(\rho D W_{\theta} \nabla \theta\right)-L e \rho D \nabla \theta \cdot \nabla W_{\theta}+\rho W W_{\theta} .
\end{gathered}
$$

Here, $\partial_{t}$ designates partial derivative with respect to time, $\mathbf{u}$ is the flow velocity vector, $W_{c}$ and $W_{\theta}$ are the first-order partial derivatives of the rate $W$ with respect to $c$ and $\theta$, respectively. Subsequently, application of a chain rule $\nabla \Omega=\Omega_{c} \nabla c+\Omega_{\theta} \nabla \theta$ to $\Omega=W, \Omega=W_{c}$, and $\Omega=W_{c}$ yields

$$
\begin{aligned}
& \rho \partial_{t} W+\rho \mathbf{u} \cdot \nabla W=\nabla \cdot(\rho D \nabla W)+(L e-1) \nabla \cdot\left(\rho D W_{\theta} \nabla \theta\right)-W_{c c} \rho D \nabla c \cdot \nabla c \\
& -W_{c \theta} \rho D \nabla c \cdot \nabla \theta-L e W_{\theta \theta} \rho D \nabla \theta \cdot \nabla \theta-L e W_{c \theta} \rho D \nabla c \cdot \nabla \theta+\rho W W_{c}+\rho W W_{\theta}
\end{aligned}
$$

where $W_{c c}, W_{c \theta}$, and $W_{\theta \theta}$ are the second-order derivatives with respect to $c$ and $c, c$ and $\theta, \theta$ and $\theta$, respectively. Finally, using the continuity equation

$$
\partial_{t} \rho+\nabla \cdot(\rho \mathbf{u})=0
$$

we arrive at

$$
\begin{gathered}
\partial_{t}(\rho W)+\nabla \cdot(\rho \mathbf{u} W)=\nabla \cdot(\rho D \nabla W)+(L e-1) \nabla \cdot\left(\rho D W_{\theta} \nabla \theta\right)-W_{c c} \rho D \nabla c \cdot \nabla c \\
-(1+L e) W_{c \theta} \rho D \nabla c \cdot \nabla \theta-L e W_{\theta \theta} \rho D \nabla \theta \cdot \nabla \theta+\rho W W_{c}+\rho W W_{\theta} \equiv T_{\Sigma} .
\end{gathered}
$$

Ensemble averaging of Eq. (7) yields

$$
\begin{gathered}
\partial_{t}(\overline{\rho W})+\underbrace{\nabla \cdot(\overline{\rho \mathbf{u} W})}_{T_{1}}=\underbrace{\nabla \cdot \overline{\rho D \nabla W}}_{T_{2}}+\underbrace{(L e-1) \nabla \cdot \overline{\rho D W_{\theta} \nabla \theta}}_{T_{3}} \underbrace{-\overline{W_{c c} \rho D \nabla c \cdot \nabla c}}_{T_{4}} \\
\underbrace{-(1+L e) \overline{W_{c \theta} \rho D \nabla c \cdot \nabla \theta}}_{T_{5}} \underbrace{-L e \overline{W_{\theta \theta} \rho D \nabla \theta \cdot \nabla \theta}}_{T_{6}}+\underbrace{\overline{\rho W W_{c}}}_{T_{7}}+\underbrace{\overline{\rho W W_{\theta}}}_{T_{8}} \equiv \overline{T_{\Sigma}} .
\end{gathered}
$$

If $L e=1$, then, the rate $W$ depends solely on $c$. Consequently, $W_{\theta}=W_{c \theta}=W_{\theta \theta}=T_{3}=T_{5}=T_{6}=T_{8}=0$ and Eqs. (7) and (8) reduce to transport equations derived and studied earlier [20-22]. In this case, terms $\bar{T}_{4}$ and $\overline{T_{7}}$ dominate in Eq. (8), i.e., their signs are opposite and their magnitudes are much larger than the magnitudes of other terms or $\left|\overline{T_{4}}+\overline{T_{7}}\right|$.

\footnotetext{
${ }^{1}$ A set of transport equations for reaction rates can also be derived in the context of complex chemistry. Such a detailed and complex study will be undertaken after thorough investigation of relatively simple cases.
} 
Accordingly, development of separate closure relations for $\overline{T_{4}}$ and $\overline{T_{7}}$ does not seem to be promising, because even small errors in evaluating any of the two terms may be large when compared to $\left|\overline{T_{1}}\right|$ or $\left|\overline{T_{\Sigma}}\right|$. To resolve the problem, a joint closure relation for $\overline{T_{2}}+\overline{T_{4}}+\overline{T_{7}}$ was developed $[20,21]$ based on studies of stretched laminar premixed flames. In the present paper, the same approach is extended to flames characterized by $L e \neq 1$. For this purpose, behavior of $T_{\Sigma}$ in stretched laminar premixed flames is analytically and numerically explored in Sect. 3. Then, results obtained from the laminar flames are utilized to develop a joint closure relation for the sum $\overline{T_{\Sigma}}$ of all terms on the RHS of Eq. (8) in turbulent flames.

\section{Stretched laminar premixed flames}

\subsection{Statement of the problem}

Following [20,21], let us consider four simple problems widely used in studies of stretched laminar premixed flames. These are (1) an expanding spherical flame, (2) an expanding cylindrical flame, (3) a steady strained flame with a cylindrical surface, and (4) a steady strained planar flame. If, in cases (3) and (4), all flame and flow characteristics with exception of the transverse (parallel to the flame surface) velocity $v$ are assumed to be constant in the transverse direction in the vicinity of the flow symmetry axis (normal to the flame surface), then, transport equations that model all four flame types can be written in the same form

$$
\frac{\partial}{\partial t}(\varrho \Phi)+\rho \varrho \Phi+\frac{1}{r^{k}} \frac{\partial}{\partial r}\left(r^{k} \varrho v \Phi\right)=\frac{1}{r^{k}} \frac{\partial}{\partial r}\left(r^{k} \varrho^{1-m} d_{\Phi} \frac{\partial \Phi}{\partial r}\right)+\varrho S_{\Phi}
$$

e.g., see Ref. [23]. Here, $\Phi=\{1, g, c, \theta\}, d_{\Phi}=\left\{1, D_{u}, D_{u}, L e D_{u}\right\}, S_{\Phi}=\left\{0,-g^{2}+J / \varrho, \varrho W, \varrho W\right\}, \varrho=\rho / \rho_{u}$ is the normalized density, $g$ is rate of strain, $k=\{0,1,2\}$ for planar, cylindrical, and spherical flames, respectively ( $g=0$ if $k=2$ ), and $m$ is a power exponent of the temperature dependence of the diffusivity $D$.

The boundary conditions read

$$
\begin{gathered}
v(0, t)=\frac{\partial g}{\partial r}(0, t)=\frac{\partial c}{\partial r}(0, t)=\frac{\partial \theta}{\partial r}(0, t)=0, \\
\frac{\partial c}{\partial r}(r \rightarrow \infty, t)=\frac{\partial \theta}{\partial r}(r \rightarrow \infty, t)=0, \quad g(r \rightarrow \infty, t)=J .
\end{gathered}
$$

\subsection{Theoretical study}

Application of a method used in Sect. 2 to Eq. (9) written for $\Phi=\{1, c, \theta\}$ yields

$$
\frac{\partial}{\partial t}(\rho W)+\rho g W+\frac{1}{r^{k}} \frac{\partial}{\partial r}\left(r^{k} \rho v W\right)=T_{\Sigma}
$$

where the term $T_{\Sigma}$ is re-written in the spherical or cylindrical coordinate framework in cases (1) or (2) and (3), respectively. Multiplication of Eq. (11) with $r^{k}$, followed by integration from $r=0$ to $\infty$ and multiplication with $R_{f}^{-k}$ results in 


$$
\frac{1}{R_{f}^{k}} \frac{d}{d t}\left(\rho_{u} R_{f}^{k} u_{c}\right)+\frac{1}{R_{f}^{k}} \int_{0}^{\infty} \rho g W r^{k} d r=\frac{1}{R_{f}^{k}} \int_{0}^{\infty} r^{k} T_{\Sigma} d r
$$

where

$$
u_{c}=\frac{1}{\rho_{u} R_{f}^{k}} \int_{0}^{\infty} r^{k} \rho W d r
$$

is the consumption velocity and $R_{f}$ is a flame coordinate associated, e.g., with the peak reaction rate [24].

Because the reaction zone is thin in a typical laminar flame, variations of the strain rate within the zone may be neglected to the leading order and, consequently, $g$ may be moved outside the integral on the Left Hand Side (LHS) of Eq. (12). Accordingly,

$$
\frac{1}{R_{f}^{k}} \int_{0}^{\infty} r^{k} T_{\Sigma} d r=\rho_{u} \frac{d u_{c}}{d t}+\left(\frac{k}{R_{f}} \frac{d R_{f}}{d t}+g_{W}\right) \rho_{u} u_{c}=\rho_{u} \frac{d u_{c}}{d t}+\rho_{u} u_{c} \dot{s}_{W}
$$

where $\dot{s}_{W}$ is the local stretch rate at the reaction zone. In weakly stretched flames commonly addressed by the theory [12-14], $u_{c} \approx S_{L}$ and, therefore, the time derivative on the RHS of Eq. (14) may be skipped. Consequently,

$$
\frac{1}{R_{f}^{k}} \int_{0}^{\infty} r^{k} T_{\Sigma} d r \approx \rho_{u} u_{c} \dot{s}_{W}
$$

Equation (15) was earlier derived and used to close the term $\overline{T_{\Sigma}}$ on the RHS of Eq. (8) in the case of Le $=1$ [20]. In Sect. 4, Eq. (15) will be adapted to close the sum $\overline{T_{\Sigma}}$ of all terms on the RHS of Eq. (8) in a turbulent flow.

\subsection{Numerical results}

To test the theoretical Eqs. (14) and (15), Eqs. (9) and (10) were numerically solved using an in-house code [23] applied earlier to various problems, e.g., see Refs. [20,21,23]. The initial conditions described a small pocket of adiabatic combustion products. The flame coordinate $R_{f}$ was obtained from a constraint of $W\left[r=R_{f}(t), t\right]=\max \{W(r, t)\}$ at each instant $t$ [24]. Because the laminar flame thickness was small and almost constant in the studied flames, computed results were weakly sensitive to the choice of $R_{f}$.

In the laminar flame simulations, all thermo-chemical characteristics were set equal to values used in the DNS discussed in Sect. 5.1. In particular, the Lewis number was set equal to $0.34,0.6$, or 0.8 , a ratio of $\rho_{u} S_{L} \delta_{t h} /($ Le $\rho D)$ was set equal to 1.75 , and

$$
W=B(1-c) \exp \left[-\frac{6 \sigma(1-\theta)}{\sigma-(\sigma-1)(1-\theta)}\right] .
$$

Here, $\sigma=\rho_{u} / \rho_{b}=5.5$ is the density ratio, and the rate constant $B$ depends on $L e$ in order to obtain desired laminar flame speed and thermal flame thickness $\delta_{t h}=1 / \max \{|\nabla \theta|\}$. The numerical results were normalized using $\rho_{u}, S_{L}$, and $\delta_{t h}$, which 
were independent of $L e$, because $B$ was tuned accordingly. The value of $L e=0.34$ is associated with very lean hydrogen-air flames, whereas the two other values of $L e$ are associated with lean $\mathrm{H}_{2} / \mathrm{CO} / \mathrm{CH}_{4} / \mathrm{O}_{2} / \mathrm{N}_{2}$ mixtures.
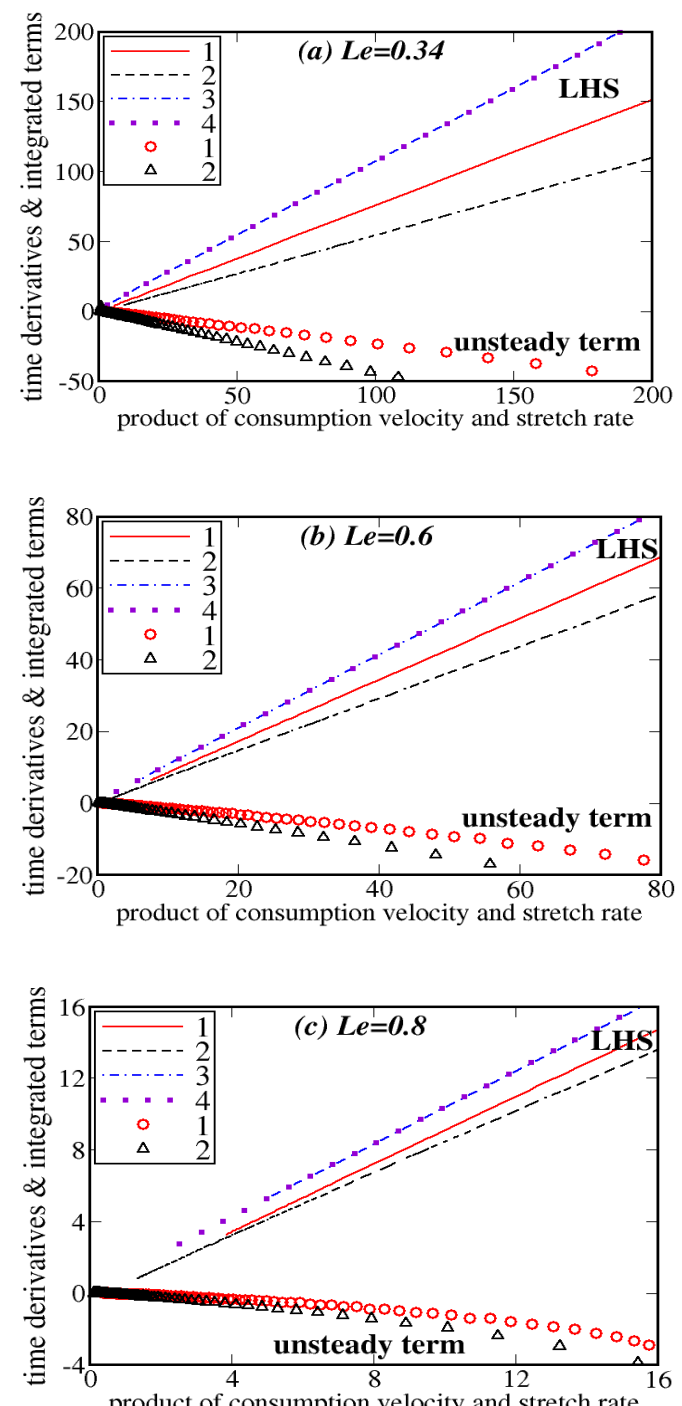

Fig. 1. The LHS of Eq. (14), see lines, and the term $\rho_{u} d u_{c} / d t$, open circles and triangles, vs. $\rho_{u} u_{c} \dot{S}_{W}$ in cases of (a) $L e=0.34$, (b) $L e=0.6$, and (c) $L e=0.8$. All quantities are normalized using $\rho_{u}, S_{L}$, and $\delta_{t h} .1$ - expanding spherical flame $(k=2, J=0), 2$ - expanding cylindrical flame $(k=1, J=0), 3$ - steady strained cylindrical flame $(k=1$, various $J), 4$ - steady strained planar flame $(k=0$, various $J)$.

Computed dependencies of the LHS of Eq. (14), see lines in Fig. 1, and the unsteady term $\rho_{u} d u_{c} / d t$, see open circles and triangles, on $\rho_{u} u_{c} \dot{s}_{W}$ (i) validate the linear Eq. (15) for variously strained steady flames, see dotted-dashed and dotted lines, and for weakly stretched $\left(u_{c} \dot{s}_{W}<S_{L}^{2} / \delta_{t h}\right)$ expanding flames, see solid and dashed lines, but (ii) show that the unsteady term $\rho_{u} d u_{c} / d t$ plays a role in highly stretched expanding flames, with the effect magnitude being increased with decreasing Le. For the goals of the present work, the most important message from Fig. 1 consists of the linear relation between the integrated term $T_{\Sigma}$, i.e., the LHS of Eq. (14), and $\rho_{u} u_{c} \dot{s}_{W}$ in a wide range of stretch rates for different flame configurations. The coefficient of proportionality is very close to unity for the stationary flames, see dotted-dashed and dotted lines, but is slightly less than unity for expanding flames at high stretch rates, see solid and dashed lines, due to the unsteady term, see open circles and triangles, on the RHS of Eq. (14). 


\section{A joint closure relation}

In a recent paper [20], Eq. (15) was theoretically and numerically obtained for stretched laminar premixed flames characterized by $L e=1$ and a simple joint closure relation

$$
\partial_{t}(\bar{\rho} \widetilde{W})+\nabla \cdot(\overline{\rho \mathbf{u} W})=\overline{T_{\Sigma}} \approx \overline{\rho W \dot{s}}=\bar{\rho} \widetilde{W}\langle\dot{s}\rangle_{W}
$$

was proposed to be used. Here, $\langle\dot{s}\rangle_{W}=\left\langle\nabla \cdot \mathbf{u}-\mathbf{n n}: \nabla \mathbf{u}+S_{d} \nabla \cdot \mathbf{n}\right\rangle_{W}$ is the stretch rate conditioned to reaction zones, $S_{d}=[\nabla \cdot(\rho D \nabla c)+\rho W] /(\rho|\nabla c|)$ is the displacement speed, $\mathbf{n}=-\nabla c /|\nabla c|$ is the unit normal vector, and $T_{3}=T_{5}=T_{6}=$ $T_{8}=0$ in $\overline{T_{\Sigma}}$ in Eq. (8) in the earlier case of $L e=1$. Subsequently, Eq. (15) was validated [20,21] against three sets of DNS data obtained earlier by two independent research groups under substantially different conditions associated either with the flamelet regime or with the thin reaction zone regime of premixed turbulent combustion [25]. All these data were computed at $L e=1$.

The physical reasoning for hypothesizing Eq. (15) at $L e=1$ were as follows [20]. As the analysis of stretched laminar flames shows that $T_{\Sigma}$ in Eq. (7) yields $\rho_{u} u_{c} \dot{s}_{W}$ after integration along the normal to a laminar flame, one may assume that $\overline{T_{\Sigma}} \propto \gamma_{r} \rho_{u} u_{c} \dot{s}_{W} / \delta_{r}$, where $\gamma_{r}$ is the probability of finding reaction zones and the reaction zone thickness $\delta_{r}$ is obtained from transformation of integration over $c$ using a probability density function $P(c)$ to integration along local normal direction to the reaction zone [20]. Similarly, $\bar{\rho} \widetilde{W} \propto \gamma_{r} \rho_{u} u_{c} / \delta_{r}$, and, consequently, $\overline{T_{\Sigma}} \propto \overline{\rho W \dot{s}}$.

Equation (15) and almost straight lines shown in Fig. 1 allow us to extend the previous analysis [20,21] invoking basically the same physical reasoning. Accordingly, based on Eq. (15) and Fig. 1, the sum $\overline{T_{\Sigma}}$ of all terms on the RHS of Eq. (8) is hypothesized to be equal to a product $\bar{\rho} \widetilde{W}\langle\dot{s}\rangle_{W}$ of the mean mass rate $\bar{\rho} \widetilde{W}$, whose transport is modeled by Eq. (8), and the stretch rate $\langle\dot{s}\rangle_{W}$ conditioned to the reaction zone, which characterizes the local flame turbulence-interaction. Consequently, the simple joint closure relation given by Eq. (17) may be applied to flames characterized by $L e<1$, with $S_{d}$ and $\mathbf{n}$ being eventually substituted with $S_{d, \theta}=[L e \nabla \cdot(\rho D \nabla \theta)+\rho W] /(\rho|\nabla \theta|)$ and $\mathbf{n}_{\theta}=-\nabla \theta /|\nabla \theta|$, respectively. It is worth stressing that the developed approach does not involve any tuning coefficient different from unity. Contrary to a usual flamelet approach, which is based on an assumption that the entire flamelets in a turbulent flow retain the structure of (stretched) laminar flames, the present approach invokes a similar hypothesis only for the reaction zone. Accordingly, the domain of validity of the present approach is expected to be wider when compared to a flamelet approach, because the reaction zone is typically thinner and is less perturbed by small-scale turbulent eddies when compared to the flamelet preheat zone. This hypothesis requires further validation in future analyses.

It is also worth noting that Eq. (17) does not solve the problem of closing the RHS of Eq. (8), because the conditioned stretch rate $\langle\dot{s}\rangle_{W}$ still requires a closure relation. Nevertheless, Eq. (17) appears to be the crucial step to solving the problem, as a sum of seven unclosed terms, which counterbalance one another almost completely, is reduced to a single term. The present approach follows the pioneering ideas by Kolmogorov [26], based on which a widely used closed transport equation for the mean rate $\bar{\varepsilon}$ of the dissipation of turbulent kinetic energy $\bar{k}$ was developed [27]. Indeed, the exact transport equation 
for $\bar{\varepsilon}$ involves two unclosed dominating terms, which have opposite signs and whose magnitude is increased by $R e_{t}$ and is unbounded at $R e_{t} \rightarrow \infty$ [28]. However, Kolmogorov [26] proposed to explore the two terms jointly and hypothesized that the sum of them was proportional to $\bar{\varepsilon}^{2} / \bar{k}$ and, therefore, was bounded at $R e_{t} \rightarrow \infty$. As stressed by Spalding [27], these pioneering ideas laid foundation of a family of two-equation models of turbulence. The joint closure relation given by Eq.

(17) follows similar ideas by also utilizing results of laminar flame studies in Sect. 3.

\section{Validation}

\subsection{DNS attributes}

Because DNS data analyzed in the following were discussed in details in various papers, e.g., see Refs. [29-33] and references quoted therein, we will restrict ourselves to a brief summary of the simulations.

The cases considered here were simulated using a well-known DNS code SENGA [34], which solved the standard governing equations of mass, momentum, energy, and combustion progress variable written in a non-dimensional form. Combustion chemistry was reduced to a single reaction, with the used thermo-chemical parameters being specified in Sect. 3.3, see Eq. (16). A cubic domain of $24.1 \delta_{t h} \times 24.1 \delta_{t h} \times 24.1 \delta_{t h}$ was discretised by a uniform Cartesian grid of $230 \times$ $230 \times 230$ points. The spatial discretisation and explicit time advancement were carried out by high-order finite-difference $\left(10^{\text {th }}\right.$ order for internal points, with the order of differentiation gradually reducing to a one-sided $2^{\text {nd }}$ order scheme at the nonperiodic boundaries) and Runge-Kutta ( $3^{\text {rd }}$ order) schemes. The boundaries in the direction of mean flame propagation (here, $x$-direction) were non-reflecting and were specified according to the Navier-Stokes Characteristic Boundary Condition scheme [35]. The transverse boundaries were periodic. The initial turbulent field was generated using a pseudo-spectral method [36] which yielded a homogeneous isotropic distribution of velocity fluctuations. At $t=0$, this turbulence field was superimposed on a steady-state unstrained laminar-flame solution. The flame-turbulence interaction evolved under decaying turbulence. Three $\left(L e=0.34,0.6\right.$, and 0.8 ) flames were studied for the initial $u^{\prime} / S_{L}=7.5$ and normalized longitudinal integral length scale $l / \delta_{t h}=2.5$. The initial Damköhler number $D a=S_{L} l /\left(\delta_{t h} u^{\prime}\right)=0.33$, Karlovitz number $K a=$ $\left(u^{\prime} / S_{L}\right)^{1.5}\left(l / \delta_{t h}\right)^{-0.5}=13$, and turbulent Reynolds number $R e_{t}=u^{\prime} l_{t} / v_{u}=113$, where $l_{t}=\bar{k}^{3 / 2} / \bar{\varepsilon}$. The simulations were continued for 3.34 initial eddy turnover times $l / u^{\prime}$. At that time, $u^{\prime} / S_{L}$ (or $\left.l / \delta_{t h}\right)$ decreased (increased) by $50 \%(70 \%)$ of the initial value.

These simulations yielded a strong increase in $S_{t} / S_{L}$ with decreasing the Lewis number $\left(S_{t} / S_{L}=2.5\right.$, 4.6, and 13.7 at $L e=0.8,0.6$, and 0.34 , respectively [29]), in line with various experimental data reviewed elsewhere [1-3]. Accordingly, the DNS data are well appropriate for the major goal of the present study.

\subsection{Results and discussion}

Figure 2 shows that, as expected, terms $\overline{T_{5}}, \overline{T_{6}}, \overline{T_{7}}$, and $\overline{T_{8}}$ dominate in Eq. (8), i.e., (i) the magnitude of each of these terms is much larger than the magnitude of another term in Eq. (8) and (ii) the positive sign of terms $\overline{T_{5}}$ and $\overline{T_{8}}$ is opposite to the negative sign of terms $\overline{T_{6}}$ and $\overline{T_{7}}$ so that $\left|\overline{T_{5}}+\overline{T_{6}}+\overline{T_{7}}+\overline{T_{8}}\right| \ll \min \left\{\left|\overline{T_{5}}\right|,\left|\overline{T_{6}}\right|,\left|\overline{T_{7}}\right|,\left|\overline{T_{8}}\right|\right\}$ in the largest part of the mean 
flame brush. Thus, Fig. 2 implies that the use of a separate closure relation for each term on the RHS of Eq. (8) may result in a large error in $\overline{T_{\Sigma}}$ due to small errors inherent to any model closure relation.
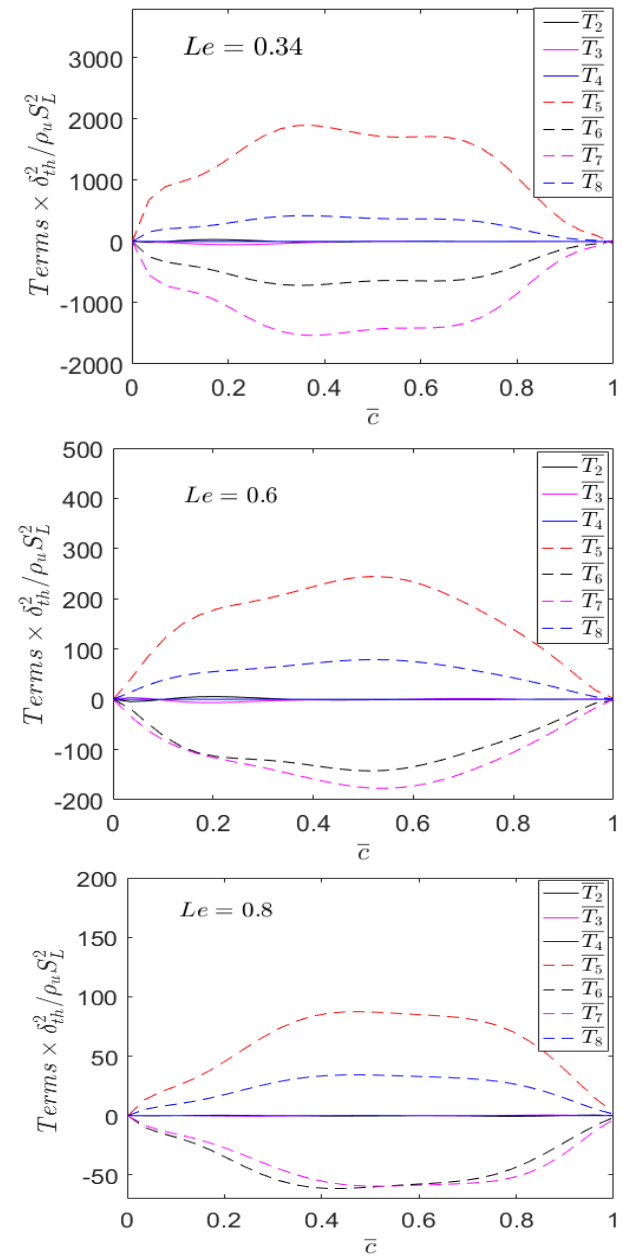

Fig. 2. Various terms on the RHS of Eq. (8), normalized using $\rho_{u}, S_{L}$, and $\delta_{t h}$.

Figure 3 shows that the joint closure relation given by Eq. (17) yields encouraging results for all three Lewis numbers. In particular, at low values of $\bar{c}$, the approach predicts a significant increase in $\overline{T_{\Sigma}}$ with decreasing Le. Moreover, the approach predicts $\overline{T_{\Sigma}}(\bar{c})$ at $L e=0.8$ and 0.6 , as well as at $\bar{c} \leq 0.3$ and $L e=0.34$. At larger values of $\bar{c}$ and $L e=0.34$, the joint closure relation overestimates $\overline{T_{\Sigma}}(\bar{c})$, probably, due to the neglect of the unsteady term on the RHS of Eq. (14) or an increase in the probability of finding negatively curved (and negatively stretched) reaction zones with increasing $\bar{c}$. Since the present analysis of stretched laminar flames has yet been restricted to planar or positively curved flames, the linear relation given by Eq. (15) may not hold if the flame is negatively curved. This issue will be addressed in future work. Nevertheless, results reported in Fig. 3 appear to be promising bearing in mind the lack of any tuning parameter in Eq. (17). It is also worth remembering that Eq. (17) was earlier validated [20,21] against three other DNS databases computed by two independent research groups under substantially different conditions, but at $L e=1$.

Figure 4 shows normalized profiles of $\langle\dot{s}\rangle_{W}=\overline{\rho W \dot{s}} / \overline{\rho W}$, obtained for various $L e$ using $S_{d, \theta}$. This figure implies that the increase in $\overline{T_{\Sigma}}$ at low $\bar{c}$ with decreasing Le, shown in Fig. 3, stems from an increase in the conditioned stretch rate. The 
increase in $\langle\dot{s}\rangle_{W}(\bar{c} \ll 1)$ stems from the fact that a flame characterized by a lower Le can survive under the influence of a higher stretch rate, with all other things being equal, e.g., cf. numbers on the abscissa axes in Figs. 1a, 1b, and 1c. For purely geometrical reasoning, positively curved reaction zones dominate at low $\bar{c}$, with the highest possible value of the local curvature being increased with decreasing $L e$, thus, making $\langle\dot{s}\rangle_{W}$ larger.
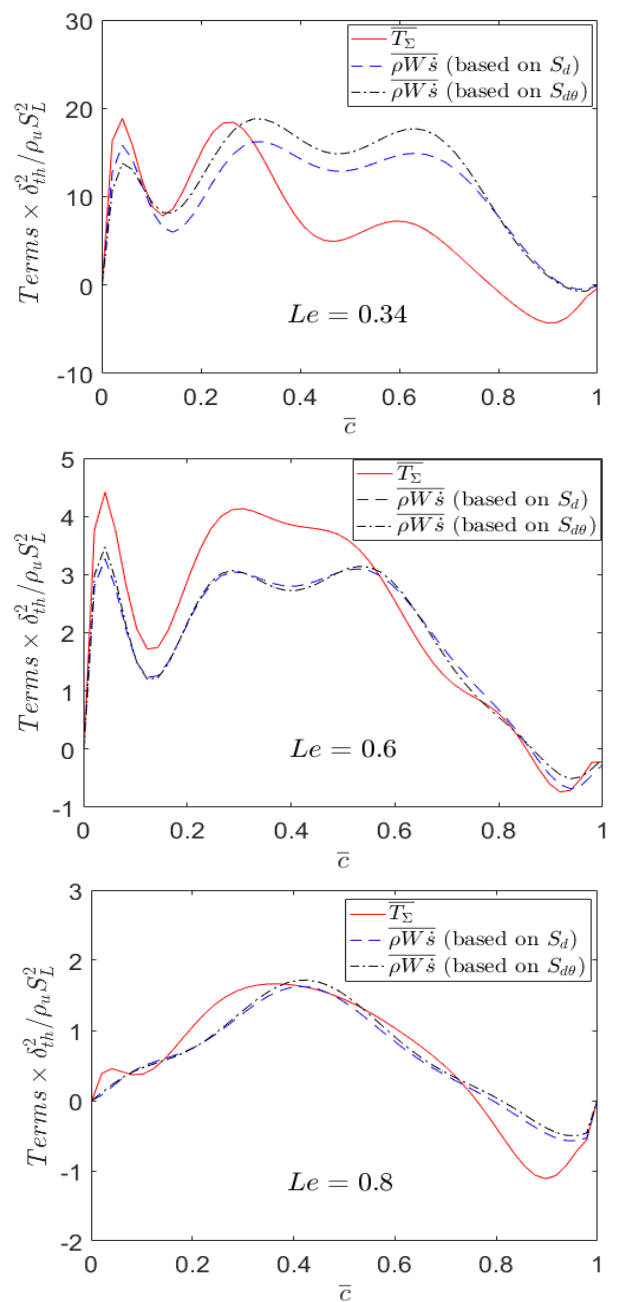

Fig. 3. Validation of Eq. (17). Dashed and dotted-dashed lines show results obtained using $S_{d}$ and $S_{d, \theta}$ when evaluating the stretch rate.

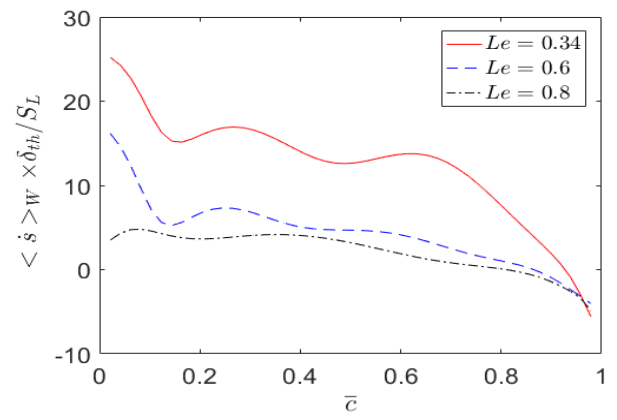

Fig. 4. Profiles of the conditioned stretch rates, obtained for various $L e$ and normalized using $S_{L}$ and $\delta_{t h}$.

Moreover, Eq. (17) and Fig. 4 provide a clue to understanding why the leading edge of a premixed turbulent flame brush can control the flame speed $S_{t}$, as hypothesized within the framework of the leading point concept put forward by Zeldovich 
[12]. The concept is discussed in details elsewhere [1-3], with the body of evidences in favor of it has been growing over the past years [9-11,37-40]. Indeed, if the turbulent flux $\overline{\rho \mathbf{u}^{\prime \prime} W^{\prime \prime}}$ is weak when compared to the mean flux $\bar{\rho} \widetilde{\mathbf{u}} \widetilde{W}$, as shown recently [22], then, for qualitative discussion, Eq. (17) may be simplified as follows

$$
\rho_{u} S_{t} \frac{d \widetilde{W}}{d x}=\bar{\rho} \widetilde{W}\langle\dot{s}\rangle_{W}
$$

for a flame that moves from right to left in a statistically 1D planar fully-developed case. The form of this equation and Fig. 4 implies that the mean rate $\widetilde{W}$ is increased with $x$ at low $\bar{c}$, where $\langle\dot{s}\rangle_{W}>0$, but is decreased with $x$ at large $\bar{c}$, where $\langle\dot{s}\rangle_{W}<$

0 . Accordingly, $\rho_{u} S_{t}=\int_{-\infty}^{\infty} \bar{\rho} \widetilde{W} d x$, i.e., the area under a curve of $\bar{\rho} \widetilde{W}(x)$, is strongly affected by the slope $d \widetilde{W} / d x$ at low $\bar{c}$. The larger the slope, the larger the area and, hence, the larger $S_{t}$. Consequently, $S_{t}$ is increased by the conditioned stretch rate $\langle\dot{s}\rangle_{W}$ evaluated near the leading edge.

For instance, if (i) the dependence of $\bar{\rho} \widetilde{W}$ on $\tilde{c}$ has a dome-like shape, e.g., $\bar{\rho} \widetilde{W} \propto \rho_{u} \bar{c}(1-\bar{c})$ [41], and (ii) $\bar{c}$ is approximated as $\bar{c}=\left[1+\exp \left(4 x / \delta_{t}\right)\right]^{-1}[3,42,43]$, where $\delta_{t}$ is the mean flame brush thickness, then, $\rho_{u} S_{t} d \widetilde{W} / d x \propto$ $\rho_{u} S_{t}(1-2 \bar{c}) d \bar{c} / d x=\rho_{u} S_{t}(1-2 \bar{c}) \bar{c}(1-\bar{c}) / \delta_{t} \propto(1-2 \bar{c})\left(S_{t} / \delta_{t}\right) \bar{\rho} \widetilde{W}$ and Eq. (18) implies that $S_{t} \propto \delta_{t}\langle\dot{s}\rangle_{W}(\bar{c} \rightarrow 0)$, i.e., the turbulent flame speed is controlled by the conditioned stretch rate at the flame leading edge. Thus, Fig. 4 and Eq. (18) explain the aforementioned strong increase in the computed values of $S_{t} / S_{L}$ with decreasing $L e\left(S_{t} / S_{L}=2.5\right.$, 4.6, and 13.7 at $L e=0.8,0.6$, and 0.34, respectively [29]). Moreover, Eq. (18) implies that modeling of the behavior of the product $\bar{\rho} \widetilde{W}\langle\dot{s}\rangle_{W}$ at low $\bar{c}$ is of paramount fundamental importance and this will be a subject for future study.

\section{Conclusions}

Transport equations for the rate $W$ of product creation and its Favre-averaged value $\widetilde{W}$ were derived by considering a premixed turbulent flame characterized by the Lewis number different from unity. Subsequently, based on theoretical and numerical investigations of variously stretched laminar flames characterized by $L e<1$, a simple joint closure relation for the sum of all seven unclosed terms on the RHS of the equation for $\widetilde{W}$ was developed, see Eq. (17). While Eq. (17) does not solve the problem of closing the RHS of the transport equation for $\widetilde{W}$, as the conditioned stretch rate $\langle\dot{s}\rangle_{W}$ still requires modeling, the proposed joint closure relation appears to be the key step to solve the problem, as a sum of seven unclosed terms, which counterbalance one another almost completely, is reduced to a single term.

The joint closure relation was assessed by analysing 3D DNS data obtained from three statistically 1D, planar, adiabatic, premixed turbulent flames in the case of a single-step chemistry and $L e=0.34,0.6$, or 0.8 . The obtained agreement between the model and DNS results is promising, thus, indicating that the sum $\bar{T}_{\Sigma}$ of seven unclosed terms on the RHS of Eq. (8), some of them counterbalance one another almost completely, may be modelled with a single term $\bar{\rho} \widetilde{W}\langle\dot{s}\rangle_{W}$. Assessment of this hypothesis at various $R e_{t}$ or $K a$ and for different flame configurations is necessary for further validation. 


\section{Acknowledgements}

AL gratefully acknowledges the financial support provided by CERC and Chalmers Area of Advance Transport. NC gratefully acknowledges the financial support provided by EPSRC, UK. VS gratefully acknowledges the financial support provided by ONERA and by the Grant of the Ministry of Education and Science of the Russian Federation (Contract No.

14.G39.31.0001 of 13.02.2017).

\section{References}

[1] Kuznetsov VR, Sabelnikov VA. Turbulence and Combustion, Hemisphere Publ Corp, New York, 1990.

[2] Lipatnikov AN, Chomiak J. Molecular transport effects on turbulent flame propagation and structure. Prog Energy Combust Sci 2005;31:1-74.

[3] Lipatnikov AN. Fundamentals of Premixed Turbulent Combustion, CRC Press, Boca Raton, Florida, 2012.

[4] Kitagawa T, Nakahara T, Maruyama K, Kado K, Hayakawa A, Kobayashi S. Turbulent burning velocities of hydrogenair premixed propagating flames at elevated pressures. Int J Hydrogen Energy 2008;33:5842-9.

[5] Yang S, Saha A, Liang W, Wu F, Law CK. Extreme role of preferential diffusion in turbulent flame propagation. Combust Flame 2018;188:498-504.

[6] Nakahara M, Kido H. Study of turbulent burning velocity of hydrogen mixtures including hydrocarbons. AIAA J 2008;46:1569-75.

[7] Muppala SRP, Nakahara M, Aluri NK, Kido H, Wen JX, Papalexandris MV. Experimental and analytical investigation of the turbulent burning velocity of two-component fuel mixtures of hydrogen, methane and propane. Int J Hydrogen Energy 2009;34:9258-65.

[8] Daniele S, Jansohn P, Mantzaras J, Boulouchos K. Turbulent flame speed for syngas at gas turbine relevant conditions. Proc Combust Inst 2011;33:2937-44.

[9] Venkateswaran P, Marshall A, Shin DH, Noble D, Seitzman J, Lieuwen T. Measurements and analysis of turbulent consumption speeds of $\mathrm{H}_{2} / \mathrm{CO}$ mixtures. Combust Flame 2011;158:1602-14.

[10] Venkateswaran P, Marshall A, Seitzman J, Lieuwen T. Scaling turbulent flame speeds of negative Markstein length fuel blends using leading points concepts. Combust Flame 2015;162:375-87.

[11] Zhang W, Wang J, Yu Q, Jin W, Zhang M, Huang Z. Investigation of the fuel effects on burning velocity and flame structure of turbulent premixed flames based on leading points concept. Combust Sci Technol, in press, available online https://www.tandfonline.com/doi/full/10.1080/00102202.2018.1451848

[12] Zeldovich YaB, Barenblatt GI, Librovich VB, Makhviladze GM. The Mathematical Theory of Combustion and Explosions, Consultants Burea, New York, 1985.

[13] Clavin P. Dynamical behavior of premixed flame fronts in laminar and turbulent flows. Prog Energy Combust Sci 1985;11:1-59.

[14] Matalon M. Intrinsic flame instabilities in premixed and nonpremixed combustion. Annu Rev Fluid Mech 2007;39:16391.

[15] Veynante D, Vervisch L. Turbulent combustion modeling. Prog Energy Combust Sci 2002;28:193-266.

[16] Poinsot T, Veynante D. Theoretical and Numerical Combustion, 2nd ed., Edwards, Philadelphia, 2005.

[17] Bilger RW, Pope SB, Bray KNC, Driscoll JF. Paradigms in turbulent combustion research. Proc Combust Inst 2005;30:21-42.

[18] Swaminathan N, Bray KNC. Eds. Turbulent Premixed Flames, Cambridge University Press, Cambridge, U.K., 2011.

[19] Echekki T, Mastorakos Ep. Eds. Turbulent Combustion Modeling, Springer, 2011.

[20] Sabelnikov VA, Lipatnikov AN, Chakraborty N, Nishiki S, Hasegawa T. A transport equation for reaction rate in turbulent flows. Phys Fluids 2016;28:081701.

[21] Sabelnikov VA, Lipatnikov AN, Chakraborty N, Nishiki S, Hasegawa T. A balance equation for the mean rate of product creation in premixed turbulent flames. Proc Combust Inst 2017;36:1893-901.

[22] Lipatnikov AN, Sabelnikov VA, Chakraborty N, Nishiki S, Hasegawa T. DNS study of closure relations for convection flux term in transport equation for mean reaction rate in turbulent flow. Flow Turbul Combust 2018;100:7592.

[23] Lipatnikov AN, Chomiak J. Lewis number effects in premixed turbulent combustion and highly perturbed laminar flames. Combust Sci Technol 1998;137:277-98.

[24] Giannakopoulos GK, Gatzoulis A, Frouzakis CE, Matalon M, Tomboulides AG. Consistent definitions of "Flame Displacement Speed" and "Markstein Length" for premixed flame propagation. Combust Flame 2015;162:1249-64.

[25] Peters N. Turbulent Combustion, Cambridge University Press, Cambridge, UK, 2000.

[26] Kolmogorov AN. Equations of turbulent motion of an incompressible fluid. Izv Akad Nauk SSSR, Physics 1942;6(12):56-8 (English translation in Proc R Soc London A 1991;434:214-6).

[27] Spalding DB. Kolmogorov's two-equation model of turbulence. Proc R Soc London A 1991;434:211-6.

[28] Monin AS, Yaglom AM. Statistical Fluid Mechanics: Mechanics of Turbulence, vol. 2, MIT Press, Cambridge, Massachusetts, 1971

[29] Chakraborty N, Cant RS. Effects of Lewis number on turbulent scalar transport and its modelling in turbulent premixed flames. Combust Flame 2009;156:1427-44.

[30] Chakraborty N, Cant RS. Effects of Lewis number on flame surface density transport in turbulent premixed combustion. Combust Flame 2011;158:1768-87. 
[31] Chakraborty N, Lipatnikov AN. Effects of Lewis number on conditional fluid velocity statistics in low Damköhler number turbulent premixed combustion: A direct numerical simulation analysis. Phys Fluids 2013;25:045101.

[32] Chakraborty N, Konstantinou I, Lipatnikov AN. Effects of Lewis number on vorticity and enstrophy transport in turbulent premixed flames. Phys Fluids 2016;28:015109.

[33] Dopazo C, Cifuentes L, Chakraborty N. Vorticity budgets in premixed combusting turbulent flows at different Lewis numbers. Phys. Fluids 2017;29:045106.

[34] Jenkins KW, Cant RS, in: Liu C, Sakell L, Beautner T. Eds. Proc. 2nd AFOSR Conf. on DNS and LES, Kluwer Academic Publishers, Dordrecht, the Netherlands, 1999, p. 192.

[35] Poinsot T, Lelef SK. Boundary conditions for direct simulations of compressible viscous flows. J Comp Phys 1992;101:104-29.

[36] Rogallo RS. Numerical Experiments in Homogeneous Turbulence, NASA Technical Memorandum 81315, NASA Ames Research Center, California, 1981.

[37] Sabelnikov VA, Lipatnikov AN. Transition from pulled to pushed premixed turbulent flames due to countergradient transport. Combust Theory Modelling 2013;17:1154-75.

[38] Sabelnikov VA, Lipatnikov AN. Transition from pulled to pushed fronts in premixed turbulent combustion: theoretical and numerical study. Combust Flame 2015;162:2893-903.

[39] Kim SH. Leading points and heat release effects in turbulent premixed flames. Proc Combust Inst 2017;36:2017-24.

[40] Dave HL, Mohan A, Chaudhuri S. Genesis and evolution of premixed flames in turbulence. Combust Flame 2018;196:386-99.

[41] Bray KNC. Studies of the turbulent burning velocity. Proc R Soc London A 1990;431:315-35.

[42] Lipatnikov AN, Chomiak J. Turbulent flame speed and thickness: phenomenology, evaluation, and application in multidimensional simulations. Prog Energy Combust Sci 2002;28:1-73.

[43] Driscoll JF. Turbulent premixed combustion: flamelet structure and its effect on turbulent burning velocities. Prog Energy Combust Sci 2008;34:91-134. 